\title{
Perfil de prescrição antibiótica no tratamento das Infecções das Vias Aéreas Superiores
}

Ana Bessa Monteiro, ${ }^{*}$ Ana Dulce Castanheira, ${ }^{* *}$ Miriam Castro, ${ }^{*}$ Nuno Capela*

\section{RESUMO}

Objectivos: Determinar a proporção de infecções das vias aéreas superiores (IVAS) tratadas com antibiótico numa Unidade de Saúde Familiar (USF); verificar quais os antibióticos mais prescritos nas IVAS; avaliar se existe relação entre a prescrição de antibióticos e a idade e o sexo do doente; avaliar se existe relação entre o antibiótico prescrito e a idade do doente.

Tipo de estudo: observacional, analítico e transversal.

Local: Unidade de Saúde Familiar (USF) Serpa Pinto, no Porto.

População: Utentes inscritos na USF com pelo menos um contacto médico codificado como IVAS.

Métodos: Recolheu-se a informação a partir dos sistemas MedicineOne ${ }^{\circledR}$ e SAM ${ }^{\circledR}$ e incluíram-se as consultas classificadas, segundo a International Classification of Primary Care-2, como: R21; R72; R74; R75; R76.

Resultados: Das 299 consultas estudadas, $61,2 \%$ foram realizadas a mulheres. A média de idades foi de 26,4 anos ( $\pm 22,3$ ). Os diagnósticos mais frequentes foram R76 $(48,1 \%)$ e R74 (38,7\%). Foi prescrito antibiótico em $68,2 \%$ das consultas, sendo a classe das penicilinas a mais prescrita $(90,6 \%)$. Não existiu relação entre a prescrição de antibióticos e o sexo ou a idade do doente. Verificou-se uma diferença estatisticamente significativa entre a idade e a classe de antibiótico prescrita: penicilinas $(25,1$ anos $\pm 22,1)$ vs restantes classes $(39,7$ anos $\pm 20,1)(p<0,001)$.

Conclusões: A classe dos beta-lactâmicos foi a mais usada para o tratamento de IVAS, com uma maior proporção de penicilinas prescritas, em relação a outros estudos. Por outro lado, verificou-se existir relação entre a idade e a classe terapêutica de antiótico prescrita.

Palavras-chave: Agentes Antibacterianos; Infecção das Vias Respiratórias Superiores; Cuidados de Saúde Primários.

\section{INTRODUÇÃO}

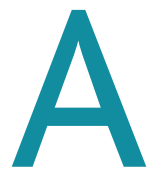
resistência aos antibióticos constitui um problema sério tanto em países desenvolvidos como em vias de desenvolvimento. Hoje em dia é geralmente aceite a relação directa entre o uso de antibióticos e a prevalência de microorganismos patogénicos resistentes entre as comunidades humanas, o que em última análise será responsável por um agravamento da mortalidade, da morbilidade, da qualidade de vida e dos custos associados à prestação de cuidados de saúde.

Por outro lado, as Infecções das Vias Aéreas Superiores (IVAS) são uma causa muito frequente de recurso aos Cuidados de Saúde Primários (CSP), sendo re-

* Interno(a) de Medicina Geral e Familiar, USF Serpa Pinto.

**Médica de Medicina Geral e Familiar, USF Serpa Pinto. portada uma frequência de 11 a 33\% de IVAS em todos os motivos de consulta de Medicina Geral e Familiar (MGF). ${ }^{1-4}$

No que diz respeito ao problema das resistências, torna-se mais grave quando consideramos o uso de antibióticos em situações para as quais não têm qualquer eficácia, como o resfriado comum, em outros síndromes virais ou quando são amplamente usados na profilaxia e não no tratamento de situações concretas.

Nos últimos anos têm-se assistido a várias acções com o intuito de sensibilizar e aprofundar conhecimentos dos profissionais de saúde e da comunidade. A avaliação recente do efeito de duas campanhas mediatizadas que decorreram nos anos de 2004 e 2005 no Nordeste de Inglaterra, e que forneceram à comunidade informação sobre o uso adequado de antibióticos, revelou um decréscimo significativo do uso de anti- 
bióticos nos meses de Inverno (menos 21,7 eventos por 1000 indivíduos, $\mathrm{p}<0,0005) .{ }^{5}$ Também nos EUA foi avaliado o impacto de um programa educacional em 21 médicos de CSP, mostrando um decréscimo de 24,6\% nas prescrições de antibióticos após o programa, reafirmando a sua importância. ${ }^{6}$

O projecto ESAC (European Surveilance of Antimicrobial Prescription), levado a cabo na Europa entre 1997 e 2002, no âmbito dos CSP, mostrou que Portugal é o 4 . $^{\circ}$ país com maior taxa de prescrição total de antibióticos em ambulatório, com uma média de cerca de $38 \%$ superior à média comunitária. ${ }^{7}$ Por outro lado verificou-se em Portugal um consumo crescente de antibióticos entre os anos de 1995 e 1999, com um acréscimo de cerca de $24 \%$ nesse período, segundo o relatório da monitorização do consumo de antibióticos do INFARMED. ${ }^{8}$

No serviço de atendimento complementar na Unidade de Saúde de Vialonga, Lisboa, foi encontrada no ano de 2000 uma elevada taxa de prescrição de antibióticos (23\% dos episódios de consulta), sendo a amigdalite aguda o diagnóstico que motivou maior prescrição de antibióticos. Os macrólidos constituíram a classe mais usada neste contexto (73 das amigdalites agudas em 93 casos). ${ }^{9}$

A rede de Médicos Sentinela estudou os actos de prescrição antibiótica notificados durante o ano de 2001. Verificou-se uma frequência de 93,2 prescrições por 1000 indivíduos, por ano, sendo mais elevada no sexo feminino ( 11.1 vs. $7.3 \%$; $\mathrm{p}<0.001$ ). Os episódios de doença do aparelho respiratório foram os mais frequentes e em $54 \%$ foi prescrita uma penicilina, seguida dos macrólidos (23,3\%) e das cefalosporinas (13,0\%). As quinolonas foram prescritas em 5,9\% dos episódios de doença do aparelho respiratório. ${ }^{4}$

Entre Dezembro de 2001 e Janeiro de 2002, o INFARMED avaliou a adequação da prescrição de antibióticos para Infecções Respiratórias em Médicos especialistas em MGF com resultados sobreponíveis ao estudo dos Médicos Sentinela quanto às classes mais prescritas. Foi encontrada elevada prescrição da associação amoxicilina com ácido clavulânico para cada uma das situações clínicas estudadas, salientando uma larga e não justificada utilização desta associação no tratamento da amigdalite e faringite. ${ }^{10} \mathrm{~A}$ inadequação da terapêutica antibiótica foi também sugerida por estudos realizados noutros países. ${ }^{11,12}$
A escassez de estudos que abordem este tema suscita a necessidade do conhecimento da adequação da prescrição antibiótica no tratamento das IVAS em CSP em Portugal.

Os objectivos deste estudo são: determinar a proporção de IVAS tratadas com antibiótico numa Unidade de Saúde Familiar do Porto (USF); verificar quais as classes fármaco-terapêuticas de antibióticos mais prescritas; avaliar se existe relação entre a prescrição de antibióticos e a idade e o sexo do doente ou entre a classe fármaco-terapêutica prescrita e a idade do doente.

\section{MÉTODOS}

Foi efectuado um estudo de tipo observacional, analítico e transversal, com a observação de 12 meses, entre 1 de Julho de 2007 e 30 de Junho de 2008, na USF Serpa Pinto, sobre a população de utentes inscritos com pelo menos um contacto médico codificado como IVAS. Uma amostra foi seleccionada de forma não aleatória consecutiva. A dimensão da amostra foi de 267 indivíduos, calculada com base na fórmula de Lehr, a partir de um valor de alfa de 0,05 , para uma prevalência estimada de IVAS tratadas com antibióticos de $50 \%$ e erro de 0,06 .

Foram incluídos os utentes que recorreram a consulta, no período em estudo, com o diagnóstico de IVAS, classificados segundo a International Classification in Primary Care - segunda edição (ICPC-2), como: sinais e sintomas da garganta (R21); infecção estreptocócica da orofaringe (R72); infecção aguda do aparelho respiratório superior (R74); sinusite (R75); amigdalite aguda (R76). As variáveis estudadas foram o sexo e a idade dos utentes, a classificação do episódio segundo ICPC2, o tratamento farmacológico com antibiótico ou outro fármaco e a classe de antibióticos prescrita.

A informação foi recolhida pelos médicos internos autores do estudo, em folha de registo de dados, a partir da listagem das consultas registadas em suporte informático - MedicineOne ${ }^{\circledR}$ - com os diagnósticos de IVAS, segundo o ICPC-2: R21; R72; R74; R75; R76. Procedeu-se posteriormente à consulta individual da folha de prescrição associada a cada episódio, caso existisse, em suporte informático Sistema Apoio Médico (SAM®), para identificação de fármacos prescritos.

Os dados recolhidos foram codificados e registados em base de dados informática - software SPSS® 11.5 - 
com determinação dos resultados referentes à estatística descritiva e à estatística inferencial. Foram utilizados os testes Qui-quadrado e T-student para comparação de proporções e para comparação de médias, respectivamente, com um nível de significância adoptado de 0,05 .

\section{RESULTADOS}

Foram analisados 299 contactos médicos classificados como IVAS, com uma média de idades dos utentes de $26,4( \pm 22,3)$ anos, sendo $61,2 \%$ do sexo feminino. Não foram documentadas alergias à penicilina.

Os episódios mais frequentemente classificados $(48,2 \%)$ foram os de R76 (quadro I). Em 5,4\% dos casos não foi prescrito qualquer fármaco antibiótico ou outro, incluindo analgésicos, antipiréticos ou anti-histamínicos. Foram prescritos antibióticos em $68,2 \%$ dos casos (IC a 95\%, 62,9 - 73,5\%), seja isoladamente como em associação a outros fármacos (quadro II). A classe de antibióticos mais utilizada foi a dos beta-lactâmicos (91,7\% quadro III).

\begin{tabular}{|c|c|}
\hline $\begin{array}{l}\text { QUADRO I. Ca } \\
\text { ICPC-2. }\end{array}$ & o dos episóc \\
\hline Código ICPC-2 & n (\%) \\
\hline R76 & $144(48,2)$ \\
\hline R74 & $116(38,8)$ \\
\hline R75 & $23 \quad(7,7)$ \\
\hline R72 & $16 \quad(5,3)$ \\
\hline R21 & 0 \\
\hline
\end{tabular}

Legenda: R76 - amigdalite aguda; R74 - infecção aguda do aparelho respiratório superior; R75 - Sinusite crónica/aguda; R72 - Infecção estreptocócica da orofaringe; R21 - Sinal/ sintoma da garganta

\begin{tabular}{lc}
\hline \multicolumn{2}{|l}{ QUADRO II. Tipo de prescrição efectuada. } \\
\begin{tabular}{lc}
\hline Tipo de prescrição efectuada & $\mathbf{n}(\%)$ \\
\hline Antibiótico & $55(18,4)$ \\
\hline Antibiótico + outro fármaco & $149(49,8)$ \\
\hline Outro fármaco & $79(26,4)$ \\
\hline
\end{tabular}
\end{tabular}

\begin{tabular}{|c|c|}
\hline Classe de antibióticos & n (\%) \\
\hline \multicolumn{2}{|l|}{ Beta-lactâmicos } \\
\hline Benzilpenicilina & $130(63,9)$ \\
\hline Amoxicilina & $37(18,1)$ \\
\hline Amoxicilina + Clavulanato & $18 \quad(8,7)$ \\
\hline Ceftriaxone & $2(1,0)$ \\
\hline \multicolumn{2}{|l|}{ Macrólidos } \\
\hline Azitromicina & $130(63,9)$ \\
\hline Claritromicina & $5 \quad(2,3)$ \\
\hline \multicolumn{2}{|l|}{ Sulfonamidas } \\
\hline Co-trimoxazol & $3(1,7)$ \\
\hline \multicolumn{2}{|l|}{ Quinolonas } \\
\hline Moxifloxacina & $1 \quad(0,7)$ \\
\hline
\end{tabular}

Não se verificou relação estatisticamente significativa entre a prescrição de antibióticos e o sexo $(\mathrm{p}=0,176)$ e a idade $(p=0,331)$. Verificou-se existir relação entre a idade e a classe terapêutica de antibióticos prescrita (quadro IV).

\section{DISCUSSÃO}

Da análise dos resultados deste estudo, verificamos que foram prescritos antibióticos na maioria dos episódios de IVAS que constituíram na sua maioria casos de amigdalite aguda. Na comunidade estas são habitualmente provocadas por agentes virais e estaria preconizado apenas o tratamento sintomático, farmacológico ou não, que foi aqui utilizado em menos casos. Tendo provavelmente sido utilizados em excesso, a classe de antibióticos mais usada foi a dos beta-lactâmicos. Verificou-se que a prescrição de antibióticos foi independente da idade e do sexo dos utentes.

O facto de a amostra ser constituída por todos os episódios de IVAS codificados naquela instituição durante um ano permitiu obviar um viés de selecção correndo, contudo, o risco de não terem sido incluídos episódios sub-codificados (viés de informação). Merece referência o facto do processo de codificação se ter iniciado na USF Serpa Pinto no ano correspondente ao período de estudo, reconhecendo-se, como tal, as dificuldades iniciais desse processo. 


\begin{tabular}{|l|c|c|c|}
\hline \multicolumn{2}{|l|}{$\begin{array}{l}\text { QUADRO IV. Relação entre a idade e a classe terapêutica de } \\
\text { antibióticos. Teste T-student. }\end{array}$} \\
\cline { 2 - 3 } & Beta-lactâmicos & $\begin{array}{c}\text { Outros } \\
\text { antibióticos }\end{array}$ & P \\
\hline $\begin{array}{l}\text { Idade em anos } \\
\text { (média } \pm \text { desvio padrão) }\end{array}$ & $25,1 \pm 22,1$ & $39,7 \pm 20,1$ & $<0,001$ \\
\hline
\end{tabular}

Por outro lado, por ser um estudo baseado na consulta da folha de receita (SAM), posteriormente ao episódio, não condicionou o perfil de prescrição médica. Consideramos que a exclusão do diagnóstico otite média aguda constitui uma limitação ao estudo.

Comparativamente aos estudos existentes na população portuguesa verificou-se uma maior percentagem de beta-lactâmicos prescritos, em relação a outras classes de antibióticos, que são terapêutica de $1 .{ }^{a}$ linha nestas situações ${ }^{10}(50,6 \%$ vs $90,6 \%)$. Os resultados obtidos traduzem-se numa mais-valia para a instituição, estímulo para a boa prática, formação contínua e satisfação para os profissionais de saúde.

As características do estudo não permitem a sua generalização a outras instituições, pelo que seriam interessantes estudos idênticos em outras unidades prestadoras de cuidados de saúde primários e mesmo em contexto hospitalar. O conhecimento do perfil de prescrição de antibióticos permitiria a identificação de lacunas/erros bem como a criação de protocolos de actuação/actualização e, com isso, um maior investimento na qualidade dos cuidados prestados.

Portanto, a classe dos beta-lactâmicos foi a mais usada para o tratamento de IVAS, com uma maior proporção de penicilinas prescritas em relação a outros estudos. Por outro lado, verificou-se existir relação entre a idade e a classe terapêutica de antibióticos prescrita.

\section{AGRADECIMENTOS}

À Dra. Clara Fonseca pela colaboração na orientação metodológica e análise estatística deste trabalho de investigação.

\section{REFERÊNCIAS BIBLIOGRÁFICAS}

1. Hofmans-Okkes IM, Lamberts $\mathrm{H}$. The International Classification of Primary Care (ICPC): new applications in research and computer-ba- sed patient records in family practice. Fam Pract 1996 Jun; 13 (3): 294-302.

2. Silva FS. Infecções agudas das vias respiratórias superiores - Estudo de novos episódios em clínica geral. Rev Port Clin Geral 1992; 9: 258-65.

3. Chan CS. What do patients expect from consultations for upper respiratory tract infections? Fam Pract 1996 Jun; 13 (3): 22935.

4. Falcão JM, Pisco AM, Simões JA, Falcão IM, Pimenta ZP, Nunes B. Prescrição de antibacterianos em clínica geral: um estudo na rede Médicos-Sentinela. Rev Port Clín Geral 2003 Jul-Ago; 19 (4): 315-29.

5. Lambert MF, Masters GA, Brent SL. Can mass media campaigns change antimicrobial prescribing? A regional evaluation study. J Antimicrob Chemother 2007 Mar; 59 (3): 537-43.

6. Juzych NS, Banerjee M, Essenmacher L, Lerner SA. Improvements in antimicrobial prescribing for treatment of upper respiratory tract infections through provider education. J Gen Intern Med 2005 Oct; 20 (10): 901-5.

7. Goossens H, Ferech M, Vander Stichele R, Elseviers M; ESAC Project Group. Outpatient antibiotic use in Europe and association with resistance: a cross-national database study. Lancet 2005 Feb 12-18; 365 (9459): 579-87.

8. Caldeira L, Alves da Silva E, Leal A, Inês M, Santos P. Relatório Interno: Utilização de Antibacterianos em Portugal. Lisboa: INFARMED; 1999.

9. Palma R. Prescrição de antibióticos no Serviço de Atendimento Complementar. Rev Port Clín Geral 2002 Jan-Fev; 18 (1): 35-52.

10. Caldeira L, Remísio E, António A, Aguair P, Fonseca A, Faria Vaz A, et al. Prescrição de antibióticos para infecções do tracto respiratório em Portugal Continental. Rev Port Clin Geral 2004 Jul-Ago; 20 (4): 417-48.

11. Leblebicioglu H, Canbaz S, Peksen Y, Gunaydin M. Physicians' antibiotic prescribing habits for upper respiratory tract infections in Turkey. J Chemother 2002 Apr; 14 (2): 181-4.

12. Gonzales R, Steiner JF, Sande MA. Antibiotic prescribing for adults with colds, upper respiratory tract infections and bronchitis by ambulatory care physicians. JAMA 1997 Sep 17; 278 (11): 901-4.

\section{CONFLITOS DE INTERESSE}

Não existe qualquer interesse financeiro a declarar pelos autores do trabalho realizado. A instituição empregadora não tem qualquer interesse ou conflito financeiro relacionado com os resultados ou conclusões do manuscrito. $O$ trabalho relatado não foi objecto de qualquer tipo de financiamento externo (incluindo bolsas de investigação).

\section{ENDEREÇO PARA CORRESPONDÊNCIA}

Nuno Filipe Borges Capela

Rua Serpa Pinto, n. ${ }^{\circ} 417,4250-466$ Porto

E-mail: nuno.capela@gmail.com

Recebido em 13/10/2010

Aceite para publicação em 26/11/2011 


\section{ABSTRACT}

\section{ANTIBIOTIC PRESCRIPTION IN UPPER RESPIRATORY TRACT INFECTIONS}

Objectives: To determine the proportion of upper respiratory tract infections (URTI) treated with antibiotics in a primary care unit, to identify the most prescribed antibiotics for the treatment of URTI, to test the association between antibiotic prescription and the age and gender of the patient, and to test the association between patient age and the antibiotic selected.

Study design: cross-sectional.

Setting: Serpa Pinto Family Health Unit (USF Serpa Pinto), Porto, Portugal.

Population: Patients registered in the Family Health Unit with at least one diagnosis of upper respiratory tract infection recorded.

Methods: information was collected from MedicineOne ${ }^{\circledR}$ and SAM ${ }^{\circledR}$ software using International Classification of Primary Care2 codes $R 21, R 72, R 74, R 75, R 76$.

Results: Of the 299 patients identified, $61.2 \%$ were women. The average age was 26.4 years $( \pm 22.3)$. The most common diagnoses were R76 (48.1\%) and R74 (38.7\%). Antibiotics were prescribed in $68.2 \%$ of visits, and penicillins were the most commonly prescribed antibiotics $(90.6 \%)$. There was no association between the prescription of antibiotics and the gender or age of the patient. There was a statistically significant association between patient age and the class of antibiotics prescribed. Penicillins were prescribed more to younger patients (mean age 25.1 years \pm 22.1 ) compared to other antibiotics (mean age 39.7 years $\pm 20.1, p<0.001$.

Conclusions: Beta-lactam antibiotics were the most commonly prescribed antibiotics for treatment of URTI, with a higher proportion of penicillins prescribed here than that found in other studies. There was an association found between age and the therapeutic class of antibiotics prescribed.

Keywords: Anti-bacterial agents; Respiratory Tract Infections; Primary Health Care. 